\title{
Evidence-Based Practice in the Health \& Social Services in China: evelopments, Strategies, Challenges
}

\author{
Feng Tong* and Yu Di Liu
}

Submission: December 20, 2017; Published: January 09, 2017

*Corresponding author: Feng Tong, Sichuan international studies university, Chong Qing, China, Tel: 17783071205; Email: viptom@foxmail.com

\begin{abstract}
For the past decade, China have made efforts to promote the development of evidence-based practice (EBP) in medicine and non-medical fields. The purpose of this article is to explore the current and the future development of EBP in health and social services in China. We first presented a brief account of EBP development in the Chinese context. Then, based on a systematic review and other literature searched, we explored empirical foundations that may support the development and advancement of EBP in China. We also elaborated on some barriers which inhibit the development of EBP, as well as proposed strategies that are in place to overcome these barriers. This article concludes with a discussion of perceived challenges to the development of EBP in health and social services in China. China has been making a great progress in economic development since 1997, however, there still have been so many social problems in the process of dramatic social changes [1]. In spite of large investments by the Chinese government to improve the well-being of the population, progress remains limited. One of the main reasons being identified for the lack of progression is that numerous decision making and practices are not based on evidence with the most up-to-date scientific data. More and more Chinese policymakers and researchers from various fields are seeking solutions from abroad. The purpose of this article is to explore the current and the future development of EBP in health and social services in China. The article will be elaborated from the development, empirical foundations, barriers which inhibit the development of EBP and challenges to the development of EBP in health and social services in China.
\end{abstract}

\section{a) Objects}

Present an analysis of the current situation of EBP in health and social services in China through evidence-based terms searching.

b) Methods

We reviewed systematic reviews of randomized controlled trials (RCTs) and other EBP literatures published by Chinese researchers in main land by January 2011.

\section{c) Search Terms}

In this research, the following terms in both English and Chinese were used to identify relevant studies: Evidence-based medicine, evidencebased practice, evidence-based education, evidence-based policy, evidence-based assessment, evidence-based management, systematic review, meta-analysis, EBP.

\section{d) Literature Sources}

All the literature search were conducted in the following sources: China Journal Full Text Database (CJFD), Chinese Scientific Journals Full Text Database (CSJD), Chinese Biomedical Literature Database (CBM), WANFANG Database, Cochrane Database of Systematic Reviews (CDSR; Issue 1, 2011), Campbell Library, Google Scholar. Additionally, the following two professional Journals were searched: Chinese Journal of Evidence-Based Medicine, China Journal of Social Work. The Chinese Journal of Evidence-Based Medicine is one of the peer review journals which guarantees the quality of the published studies.

\section{e) Inclusive Criteria}

1. Systematic review (Meta-Analysis) in health and social services written by authors from mainland China, but not only mean of studies in China.

2. Other literature named in EBP (e.g. quasi-experimental studies, expert comments).

3. Languages limited to Chinese and English.

\section{Search Results}

In the Chinese databases, 895 items, 730 items, and 245 items as well as 89 items were identified in the China Journal Full Text Database, Chinese Scientific Journals Full Text Database, Chinese Biomedical Literature Database and WANFANG, separately. In the English sources searched for Chinese authors' articles, 97 reviews, 126 protocols, 199 titles were found in the Cochrane Database of Systematic Review; Only 1 protocol was found in the Campbell Library, which was about communication education for 
older adults with hearing impairment [2]. Totally 340 items were found in the Chinese Journal of Evidence-Based Medicine; while no systematic review was identified in the China Journal of Social Work. There were 1678 titles found in the Google Scholar. In the four main Chinese databases, we found 18 non-medical metaanalyses based on 15 RCTs and 92 quasi-experimental studies, and 167 expert comments on EBP in non-medical arena (e.g. education, management). The 18 non-medical meta-analyses were composed of 7 RCTs and 40 non-RCTs (Chinese trials) and 8 RCTs and 52 non-RCTs (studies from other countries). Chinese RCTs accounted for only $6.5 \%$ in the 18 meta-analyses (Figure 1).

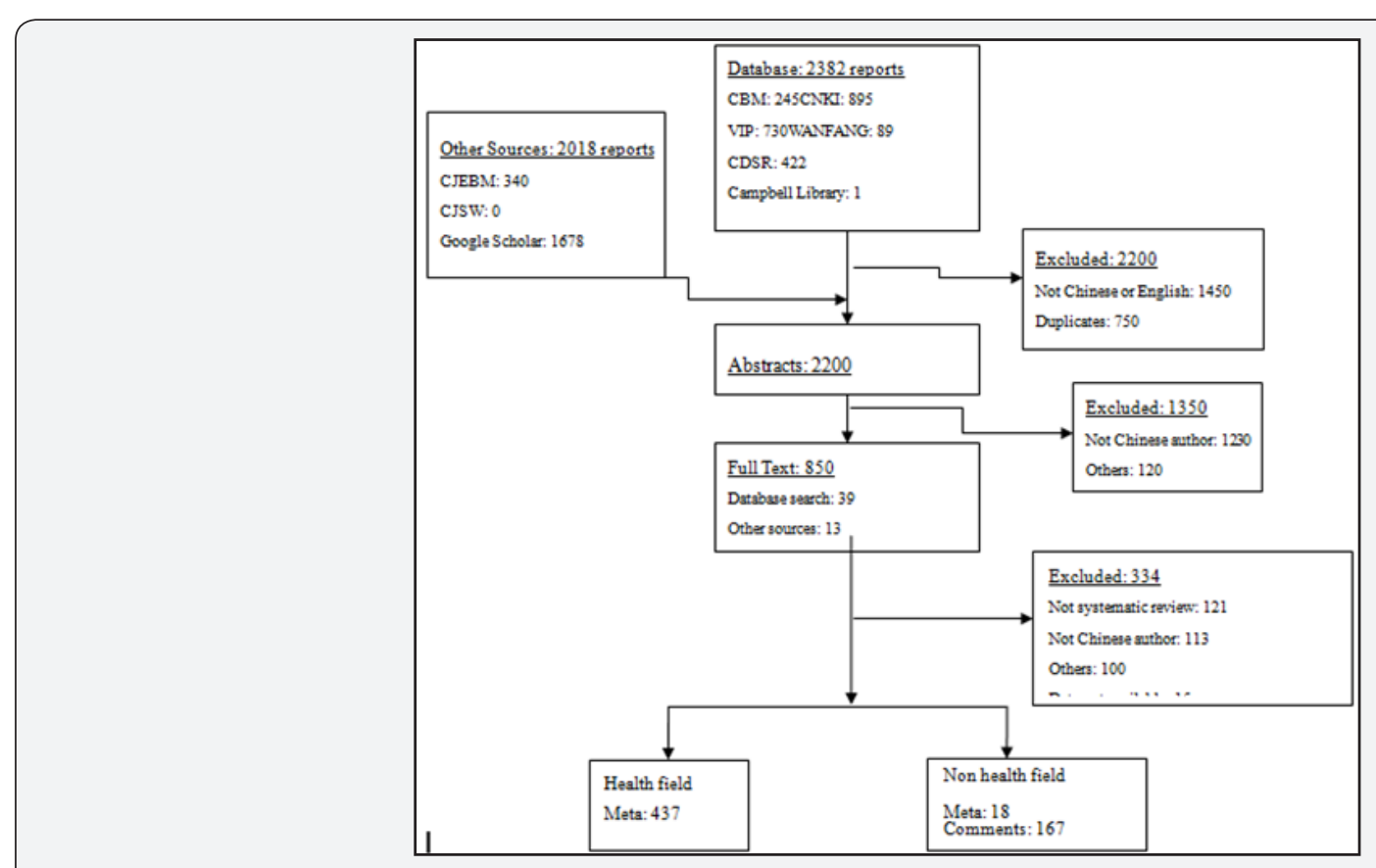

Figure 1: Literature Searching Flowing Charts.

\section{Analysis}

Using the terms systematic review, meta-analysis, evidencebased, we searched the Chinese Journal of Evidence-Based Medicine (2001-2012) and the CDSR (Issue 3, 2012). A total of 340 meta-analyses of RCTs were identified in the Chinese Journal of Evidence-Based Medicine. The published studies met the basic principles of systematic review in terms of being systematically literature searching, transparently study quality assessment, and critically inclusion and exclusion criteria making. Although the number of Cochrane systematic reviews (CSRs) in terms of full-text and protocols showed an increased annual trend, the publication rate and quality of CSRs' were uneven in the different provinces of China because of the different understanding and the inconsistently attitude to the EBP [3]. Using the search terms beginning with "evidence-based" (see "Search Terms") systematic review, meta-analysis, we searched the published literature on EBP in the four main Chinese databases and the Campbell Library, as well as the China Journal of Social Work. All 18 meta-analyses belonged to the poor quality evidence according to the AMSTAR criteria, because most of the 18 meta-analyses only used the meta-analysis statistic software in computing the included trials but not systematically and transparently reported the literature search process, as well as not assessed objectively the quality of include trials [4]. All of the included studies lack of the details of trials' design, implementation and reporting. Only
1 protocol registered by Chinese authors in the Campbell Library was identified, which was about communication education for hearing impairment [2].

\section{Discussion}

In terms of the number of difference between the literatures found in health fields and non-health fields, the EBP development in non-health fields lags behind the EBP development in health in China. Though Evidence-based Practice in health care arena developed quickly comparing with other human services in China, there are still many challenges existed. What kinds of strategies have been implemented to promote and disseminate EBM in China? Firstly, conducting more high quality trials in health and social service fields and then creating objective, and transparent evidence scheme and making decision based on current best evidence. Finally, to use and produce data, evaluate and improve interventions. Although EBP is a young subject, it narrows the gap between the knowledge production and application. EBP in other social services has begun its first step in China, but how to assist the EBP development in health and social services in China? To begin with, relying on professional researchers based on scientific and practical, and encouraging schools or relevant institutions to support the development of Evidence-based policy. Moreover, research projects and produced evidence must be appraised through scientific reliability ratting and relevance feasibility ratting by relevant experts' council. This study only 
focused on the development, strategies and challenges of EBP development in the health and social work fields in terms of SR or other EBP literatures publication in mainland China, so it is not mean that the EBP was not appropriate in China because of currently limited studies publicized. Also, this study not included the study happened in Hong Kong tom, Singapore, or other areas with more Chinese population. Both above limitations should be caution and overcome in future work.

\section{References}

1. WHO (2010) Situation analysis for health at work and development of the global working life.

This work is licensed under Creative Commons Attribution 4.0 Licens

DOI: 10.19080/GJIDD.2018.04.555630
2. Zhg Bai, Ic Kh Yang, Yp Li, Xp Shen, R Zen (2012) Communication enhancement for older adults with hearing impairment.

3. Jia Wq, Yang Kh, Tian Jh, Zhang P, Ma B, et al. (2009) Development of China's Evidence Based Medicine through the Cochrane Systematic Reviews. Chinese Journal of Evidence Based Medicine 9: 635-639.

4. Shea Bj, Gj Wells Ga, Boers M, Andersson N, Hamel C, et al. (2007) Development of AMSTAR: A measurement tool to assess the methodological quality of systematic reviews. BMC Med Res Methodol 10.

\section{Your next submission with Juniper Publishers will reach you the below assets}

- Quality Editorial service

- Swift Peer Review

- Reprints availability

- E-prints Service

- Manuscript Podcast for convenient understanding

- Global attainment for your research

- Manuscript accessibility in different formats

( Pdf, E-pub, Full Text, Audio)

- Unceasing customer service

Track the below URL for one-step submission https://juniperpublishers.com/online-submission.php 